Pacific Journal of Mathematics

THE FLAT CAUCHY PROBLEM FOR RADIALLY
HYPERBOLIC OPERATORS FROM A CHARACTERISTIC
MANIFOLD OF HIGH CODIMENSION

CARL Norman MUtChL 


\title{
THE FLAT CAUCHY PROBLEM FOR RADIALLY HYPERBOLIC OPERATORS FROM A CHARACTERISTIC MANIFOLD OF HIGH CODIMENSION
}

\author{
CARL N. MUtCHLER
}

We consider the flat Cauchy problem from a characteristic submanifold $\Sigma$ of high codimension (greater than 1).

Introduction. In the case of a characteristic hypersurface (codimension 1), several have studied the flat Cauchy problem for operators of "Fuchsian type". Baouendi and Goulaouic [4] considered the case where the operator had coefficients analytic in the space variable. In the $C^{\infty}$ case, $S$. Alinhac [1], [2] studied hyperbolic Fuchsian operators. He showed that when the operator was strictly hyperbolic for $t>0$, one had wellposedness in the flat Cauchy problem. Uniqueness results for a more general class of Fuchsian operators have been obtained by G. Roberts [8] when the coefficients are smooth.

For submanifolds of higher codimension, Alinhac and Baouendi [3] studied the uniqueness question. In particular, they defined (strictly) radially hyperbolic operators, and, when $\Sigma$ was simply a point, proved uniqueness for such operators if the solution to $P u=0$ was sufficiently flat at $t=0$. They showed the same result for second order (strictly) radially hyperbolic operators when $\Sigma$ had dimension greater than or equal to 1 . We present here results of existence, regularity, and flatness for those two cases and use their uniqueness results to obtain well-posedness in the flat Cauchy problem.

When $\Sigma$ is just a point, Theorem 3 proves well-posedness for (strictly) radially hyperbolic operators of any order. Using pseudodifferential calculus on a compact manifold without boundary, Theorem 1 shows existence of "nice" distribution solutions to the equation $P u=f$. This is done via an energy method involving Sobolev norms. For a good review of these notions, the reader is referred to Alinhac [2], Hörmander [5], Nirenberg [6], [7], and Treves [9]. Theorem 2 gives better regularity to the solutions of Theorem 1; it provides solutions flat to any finite order desired. Theorem 3 then follows from Theorem 2 and the uniqueness [3]. 
When $\operatorname{dim} \Sigma \geq 1$, Theorems 4 and 5 yield the same well-posedness result for second order (strictly) radially hyperbolic operators and similar first order hyperbolic systems respectively.

1. Results for radially hyperbolic operators when $\Sigma$ is a point. Let $W=\left\{x \in \mathbf{R}^{n}:|x| \leq T\right\}$ with $0<T \leq \infty$. We use the notation

$$
\partial_{x}^{\alpha}=\left(\frac{\partial}{\partial x_{1}}\right)^{\alpha_{1}} \cdots\left(\frac{\partial}{\partial x_{n}}\right)^{\alpha_{n}}, \quad D_{x}^{\alpha}=(-i)^{|\alpha|} \partial_{x}^{\alpha}
$$

for a multi-index $\alpha \in \mathbf{N}^{n},|\alpha|=\alpha_{1}+\cdots+\alpha_{n}$, and assume $P\left(x, \partial_{x}\right)$ to be an $m$ th order linear partial differential operator with smooth coefficients defined in $W$. We further assume that in polar coordinates $(t, \theta) \in[0, T]$ $\times S^{n-1}, x=t \theta, t=|x|$, our operator $P$ takes the form

$$
P\left(t, \theta, t \partial_{t}, \partial_{\theta}\right)=\left(t \partial_{t}\right)^{m}+\sum_{j=0}^{m-1} P_{m-j}\left(t, \theta, \partial_{\theta}\right)\left(t \partial_{t}\right)^{j},
$$

where $P_{m-j}$ is a linear partial differential operator defined on the sphere $S^{n-1}$, of order $m-j$, depending smoothly on the parameter $t$. Let $\tau$ be the dual variable to $t$ and $(\theta, \eta)$ be the variable in $T^{*}\left(S^{n-1}\right)$. For $\rho=t \tau$ and $p_{m-j}(t, \theta, \eta)$ denoting the (globally defined) principal symbol of $P_{m-j}\left(t, \theta, \partial_{\theta}\right)$, we say that $P$ of the form (1.1) is (strictly) radially hyperbolic at the point $0 \in W$ if its principal symbol, namely

$$
p_{m}(t, \theta, \rho, \eta)=(i \rho)^{m}+\sum_{j=0}^{m-1} p_{m-j}(t, \theta, \eta)(i \rho)^{J},
$$

has (distinct) real roots in $\rho$, i.e.

$$
p_{m}=i^{m} \prod_{k=1}^{m}\left(\rho-l_{k}(t, \theta, \eta)\right)
$$

with the $l_{k}$ 's real (and distinct) for all $(t, \theta, \eta) \in[0, T] \times\left(T^{*}\left(S^{n-1}\right) \backslash 0\right)$.

REMARK. If $P$ is radially hyperbolic at the point $0 \in W$, then it is hyperbolic with respect to the hypersurface $\{t=\varepsilon\}$ for each $\varepsilon, 0<\varepsilon<T$, but at the point $0 \in W, P$ becomes characteristic, i.e. for $t=0, \tau=1$, $\eta=0$, we see that $p_{m}$ vanishes.

EXAMPLE. The following operator, with $a_{1}, a_{2}$, and $a_{3}$ smooth near $(0,0) \in \mathbf{R}^{2}$, is characteristic at $(0,0)$ :

$$
\begin{aligned}
P= & \left(x^{2}-y^{2}\right)\left(\partial_{x}^{2}-\partial_{y}^{2}\right)+4 x y \partial_{x} \partial_{y}+a_{1}(x, y) x \partial_{x} \\
& +a_{2}(x, y) y \partial_{y}+a_{3}(x, y) .
\end{aligned}
$$


But in polar coordinates we have

$$
P=\left(t \partial_{t}\right)^{2}-\partial_{\theta}^{2}+a(t, \theta) t \partial_{t}+b(t, \theta) \partial_{\theta}+c(t, \theta),
$$

and hence $P$ is strictly radially hyperbolic at $(0,0) \in \mathbf{R}^{2}$.

The main result of this section is Theorem 3, which solves the flat Cauchy problem for radially hyperbolic operators at a point. The problem in this case is

$$
\left\{\begin{array}{l}
P u=f, \quad f \in C^{\infty}\left([0, T] \times S^{n-1}\right),\left.\quad \partial_{t}^{j} f\right|_{t=0}=0 \quad \text { for all } j \in \mathbf{N} \\
u \in C^{\infty}\left([0, T] \times S^{n-1}\right),\left.\quad \partial_{t}^{j} u\right|_{t=0}=0 \quad \text { for all } j \in \mathbf{N} .
\end{array}\right.
$$

As a prelude to Theorem 3, we first prove existence of somewhat "nice" distribution solutions to $P u=f$. We shall use the notation $u \in L_{-k}^{2}$ to mean $t^{-k} u \in L^{2}$ for $k$ a positive integer.

THEOREM 1. Let $M$ be a smooth compact $(n-1)$ dimensional manifold without boundary, and let $s$ and $k$ be arbitrary positive integers. Assume $P$ of the form (1.1) satisfies (1.2) with $M$ replacing $S^{n-1}$. Assume too that for $0<T<\infty, f \in C^{\infty}\left([0, T] ; H_{s}(M)\right)$ where $H_{s}(M)$ denotes the usual Sobolev space on $M$, and that $f$ is flat at $t=0$, i.e. $\left.\partial_{t}^{j} f\right|_{t=0}=0$ for all $j \in \mathbf{N}$. Then there exists $u_{s, k} \in L_{-k}^{2}\left([0, T] ; H_{s}(M)\right)$ such that $P u_{s, k}=f$ in $\mathscr{D}^{\prime}((0, T) \times M)$.

Theorem 1 is not proved by solving directly the equation $P u=f$. Instead, we introduce the new operator $Q_{\lambda}=P\left(t, \theta, t \partial_{t}+\lambda, \partial_{\theta}\right)$ for $\lambda \in \mathbf{N}$ and solve $Q_{\lambda} w=t^{-\lambda} f, \lambda$ large. In doing so, since $Q_{\lambda}\left(t^{-\lambda} v\right)=t^{-\lambda} P v$, we have a solution of the form $u=t^{\lambda} w$, i.e.

$$
P u=P\left(t^{\lambda} w\right)=t^{\lambda} Q_{\lambda} w=t^{\lambda} t^{-\lambda} f=f .
$$

To solve $Q_{\lambda} w=t^{-\lambda} f$ we use the Riesz Theorem and the following lemma concerning the adjoint operator $Q_{\lambda}^{*}$.

LEMMA 1. With the assumptions as in Theorem 1, there exists $\lambda_{s} \in \mathbf{N}$ such that for every $\lambda \geq \lambda_{s}$ and all $v \in C_{0}^{\infty}((0, T) \times M)$ we have

$$
\|v\|_{L^{2}\left([0, T] ; H_{-s}(M)\right)} \leq\left\|Q_{\lambda}^{*} v\right\|_{L^{2}\left([0, T] ; H_{-s}(M)\right)} .
$$

The preceding lemma utilizes pseudodifferential calculus by factoring the operator $Q_{\lambda}$. For each root $l_{k}(t, \theta, \eta)$ we have the corresponding factor

$$
\Lambda_{k}+\lambda=t \partial_{t}+\lambda-L_{k}\left(t, \theta, D_{\theta}\right)
$$


where $L_{k} \in C^{\infty}\left([0, T] ; \mathscr{L}^{1}(M)\right)$ is a pseudodifferential operator of order 1 on $M$, smooth in $t$, with principal symbol equal to $i l_{k}(t, \theta, \eta)$. The inequality for $Q_{\lambda}^{*}$ is shown to follow from similar inequalities for each of its factors.

If we assume more regularity in the right-hand side function $f$, the following theorem then shows us we can expect more regularity in the solution.

THEOREM 2. In addition to the assumptions of Theorem 1, let $f$ be in $C^{\infty}([0, T] \times M)$. Then for $T_{0} \in(0, T)$, there exists $u_{s, k}$ in $C^{k}\left(\left[0, T_{0}\right] ; H_{s}(M)\right)$ such that

$$
P u_{s, k}=f \quad \text { in }\left[0, T_{0}\right] \times M \quad \text { and }\left.\quad \partial_{t}^{j} u_{s, k}\right|_{t=0}=0 \quad \text { for } 0 \leq j \leq k .
$$

Theorem 2 is a consequence of Theorem 1, Sobolev's Embedding Theorem, and the following lemma.

LEMMA 2. With assumptions as in Theorem 2, let $s^{\prime}, r$, and $l^{\prime}$ be positive integers such that $s^{\prime} \geq l^{\prime}+m-1$ and $r \geq l^{\prime}+m-1$. Then there exists $u_{s^{\prime}, l^{\prime}, r} \in L_{-r}^{2}\left([0, T] ; H_{s^{\prime}}(M)\right)$ satisfying

$$
\left\{\begin{array}{l}
P u_{s^{\prime}, l^{\prime}, r}=f \text { in } \mathscr{D}^{\prime}((0, T) \times M), \\
\partial_{t}^{j} u_{s^{\prime}, l^{\prime}, r} \in L_{-(r-m-j+1)}^{2}\left([0, T] ; H_{s^{\prime}-m-J+1}(M)\right), \quad 0 \leq j \leq l^{\prime} .
\end{array}\right.
$$

Combining Theorem 2 with the uniqueness result of Alinhac and Baouendi (Theorem 2 of [3]) yields Theorem 3 below.

THEOREM 3. Let $M$ be as before and assume $P\left(t, \theta, t \partial_{t}, \partial_{\theta}\right)$ is of the form (1.1) and satisfies (1.2) with $S^{n-1}$ replaced by $M$. If

$$
f \in C^{\infty}([0, T] \times M), \quad 0<T \leq \infty,
$$

and flat at $t=0$, then there exists a unique $u \in C^{\infty}([0, T] \times M)$ such that

$$
P u=f \text { in }[0, T] \times M \quad \text { and } \quad u \text { is flat at } t=0 \text {. }
$$

REMARK. The uniqueness result proved by Alinhac and Baouendi [3] is stated as one for flat functions; however, as indicated by their proof for the hyperbolic case, functions flat to some finite order will suffice. This is exactly what our Theorem 2 provides. 
Corollary 3. Assume that $P$ has the form (1.1) and that its principal symbol has only real roots in $\rho$ of constant multiplicity, i.e.

$$
p_{m}=i^{m} \prod_{j=1}^{r}\left(\rho-l_{j}(t, \theta, \eta)\right)^{m_{j}}
$$

with the $l_{j} s(j=1, \ldots, r)$ real and distinct.

Also assume that $P$ satisfies the following Levi-type condition: For each $j=1, \ldots, r, P$ can be written

$$
P=\sum_{k=0}^{m_{J}} Q_{j, k}\left(t, \theta, t \partial_{t}, \partial_{\theta}\right) \Lambda_{J}^{m_{J}-k},
$$

with $Q_{j, k}$ a pseudodifferential operator on $(0, T) \times M$, differential in $t \partial_{t}$, of total order $m-m_{j}$, where $\Lambda_{j}=t \partial_{t}-L_{j}$ is defined as before. Then the conclusion of Theorem 3 remains true.

The Levi-type condition implies a factorization for $Q_{\lambda}$ similar to the one in the strictly hyperbolic case. This factorization also shows that the uniqueness result of Alinhac and Baouendi [3] still holds.

2. Results for radially hyperbolic operators when $\operatorname{dim} \Sigma \geq 1$. With $\Sigma$ a $\nu$-dimensional submanifold of $\mathbf{R}^{n+\nu}$ containing the origin $(\nu \geq 1)$, and $P\left(z, \partial_{z}\right), z \in \mathbf{R}^{n+\nu}$, an $m$ th order linear partial differential operator with smooth coefficients defined near $0 \in \mathbf{R}^{n+\nu}$, we assume there exists local coordinates $(x, y)$ near 0 so that $\Sigma=\{y=0\}$ and $P$, written in cylindrical coordinates $t=|y|, y=t \theta, \theta \in S^{n-1}$, takes the form

$$
P=\left(t \partial_{t}\right)^{m}+\sum_{j=0}^{m-1} P_{m-j}\left(t, x, \theta, t \partial_{x}, \partial_{\theta}\right)\left(t \partial_{t}\right)^{j}
$$

Here

$$
P_{m-j}=\sum_{|\alpha| \leq m-j} Q_{m-j-|\alpha|}^{\alpha}\left(t, x, \theta, \partial_{\theta}\right)\left(t \partial_{x}\right)^{\alpha},
$$

with $Q_{m-J-|\alpha|}^{\alpha}$ a differential operator defined on the sphere $S^{n-1}$, of order $m-j-|\alpha|$, depending smoothly on $(t, x) \in[0, T] \times \Omega, 0<T \leq \infty, \Omega$ some open set in $\mathbf{R}^{\nu}$ containing the origin.

We let $\tau, \xi$ be the dual variables to $t, x$ respectively and $(\theta, \eta)$ the variable in $T^{*}\left(S^{n-1}\right)$. Denoting the principal symbol of $Q_{m-j-|\alpha|}^{\alpha}$ by $q_{m-j-|\alpha|}^{\alpha}$ and setting $\rho=t \tau, \zeta=t \xi$, we say that the operator $P$ of the form (2.1) is (strictly) radially hyperbolic with respect to $\Sigma$ near 0 if its principal 
symbol,

$p_{m}(t, x, \theta, \rho, \zeta, \eta)=(i \rho)^{m}+\sum_{j=0}^{m-1} \sum_{|\alpha| \leq m-j} q_{m-\jmath-|\alpha|}^{\alpha}(t, x, \theta, \eta)(i \zeta)^{\alpha}(i \rho)^{\jmath}$, has (distinct) real roots in $\rho$, i.e.

$$
p_{m}=i^{m} \prod_{k=1}^{m}\left(\rho-l_{k}(t, x, \theta, \zeta, \eta)\right),
$$

with the $l_{k}$ 's real (and distinct)

for all $(t, x, \theta, \zeta, \eta) \in[0, T] \times\left(T^{*}\left(\Omega \times S^{n-1}\right) \backslash 0\right)$.

REMARK. If $P$ is radially hyperbolic with respect to $\Sigma$ near 0 , then for each $\varepsilon, 0<\varepsilon<T$, it is hyperbolic with respect to the cylinder $\{t=\varepsilon\}$, but is characteristic with respect to $\Sigma$.

EXAMPLE. The operator in $\mathbf{R}^{3}$,

$$
P=\left(y^{2}-z^{2}\right)\left(\partial_{y}^{2}-\partial_{z}^{2}\right)+4 y z \partial_{y} \partial_{z}-\left(y^{2}+z^{2}\right) \partial_{x}^{2}+2\left(y \partial_{y}+z \partial_{z}\right),
$$

with $\Sigma=\{y=z=0\}$ becomes in cylindrical coordinates

$$
P=\left(t \partial_{t}\right)^{2}-t^{2} \partial_{x}^{2}-\partial_{\theta}^{2},
$$

and hence, is strictly radially hyperbolic with respect to $\Sigma$ near $(0,0,0) \in$ $\mathbf{R}^{3}$.

The following theorem solves the flat Cauchy problem for second order operators which are radially hyperbolic with respect to manifolds of any dimension. The question regarding higher order operators is currently under study.

THEOREM 4. Let $M$ be a smooth compact $(n-1)$ dimensional manifold without boundary, and let $f \in C^{\infty}([0, T] \times \Omega \times M)$ where $0<T \leq \infty$ and $\Omega$ is some open neighborhood of the origin in $\mathbf{R}^{\nu}$. Assume $P$ has the form (2.1) with $m=2$ and satisfies (2.2) with $M$ replacing $S^{n-1}$. Then if $f$ is flat at $t=0$ there exists a unique $u \in C^{\infty}([0, T] \times \Omega \times M)$ such that

$$
P u=f \text { in }[0, T] \times \Omega \times M \quad \text { and } u \text { is flat at } t=0 \text {. }
$$

Theorem 4 is proved by obtaining an estimate similar to the one of Lemma 1 (although the techniques are different) and uses another uniqueness result due to Alinhac and Baouendi (Theorem 4' of [3]). Finally, we have the same result for certain first order hyperbolic systems.

THEOREM 5. With $M, T$, and $\Omega$ as before, let

$$
L\left(t, x, \theta, t \partial_{t}, t \partial_{x}, D_{\theta}\right)=t \partial_{t}+\sum A_{i}(t, x, \theta) t \partial_{x_{i}}+\Lambda\left(t, x, \theta, D_{\theta}\right)
$$


be a first order operator defined on $[0, T] \times \Omega \times M$ with each $A_{i}$ a smooth $N \times N$ hermitian matrix, and $\Lambda$ an $N \times N$ matrix of operators in $\mathscr{L}^{1}(M)$ depending smoothly on $(t, x) \in[0, T] \times \Omega$. Assume that the principal symbol (matrix) of $\Lambda$, defined now by $\sigma_{1}(\Lambda)$, satisfies $\sigma_{1}(\Lambda)=-\sigma_{1}\left(\Lambda^{*}\right)$. Then, given $f \in C^{\infty}([0, T] \times \Omega \times M)$, f flat at $t=0$, we have existence of $a$ unique $u \in C^{\infty}([0, T] \times \Omega \times M)$ satisfying

$$
L u=f \text { in }[0, T] \times \Omega \times M \quad \text { and } \quad u \text { is flat at } t=0 \text {. }
$$

3. Proof of Theorem 1. We begin by proving Lemma 1. Since the principal symbol of $P$ satisfies (1.2), each root $l_{k}(t, \theta, \eta)$ is positively homogeneous of degree one with respect to the fiber variable $\eta$ and smooth in $t$ and $\theta$. We let $L_{k}\left(t, \theta, D_{\theta}\right)$ be a pseudodifferential operator of order 1 on $M$, smooth in $t$, with principal symbol equal to $i l_{k}$, and set $\Lambda_{k}=t \partial_{t}-L_{k}$. From (1.1) and (1.2) one can easily check that

$$
P=\Lambda_{1} \Lambda_{2} \cdots \Lambda_{m}+\sum_{j=0}^{m-1}\left(t \partial_{t}\right)^{j} O(m-j),
$$

where $O(j)$ will be used to denote various pseudodifferential operators on $M$, of order $j$, smooth in $t$. For $k \neq j$, we know by (1.2) that $L_{k}-L_{j}$ is elliptic, so there exists a parametrix $Q_{k, j}$ of order -1 on $M$, smooth in $t$, such that $\left(L_{k}-L_{j}\right) Q_{k, j}=1 \bmod O(-1)$. Applying this fact repeatedly in (3.1) yields

$$
P=\Lambda_{1} \cdots \Lambda_{m}+\sum_{j=0}^{m-1}\left(t \partial_{t}\right)^{j} \sum_{|I| \leq m-j} \Lambda_{I} \tilde{a}_{I}^{0}
$$

where

$$
\left\{\begin{array}{l}
I=\left(i_{1}, \ldots, i_{k}\right) \subset(1, \ldots, m), \quad k \leq m, \\
\Lambda_{I}=1, \quad \text { if }|I|=0, \\
\Lambda_{I}=\Lambda_{i_{1}} \Lambda_{i_{2}} \cdots \Lambda_{i_{k}}, \quad \text { if }|I|=k, \tilde{a}_{I}^{0} \in C^{\infty}\left([0, T] ; \mathscr{L}^{0}(M)\right) .
\end{array}\right.
$$

The existence of $Q_{k, j}$ also shows that $\left(t \partial_{t}\right)^{j}=\sum_{|J| \leq j} \Lambda_{J} b_{J}^{0}$, and so

$$
P=\Lambda_{1} \cdots \Lambda_{m}+\sum_{|I| \leq m-1} \Lambda_{I} a_{I}^{0}, \quad a_{I}^{0} \in C^{\infty}\left([0, T] ; \mathscr{L}^{0}(M)\right) .
$$

With $Q_{\lambda}=P\left(t, \theta, t \partial_{t}+\lambda, \partial_{\theta}\right)$, we immediately get the factorization for the adjoint operator

$$
Q_{\lambda}^{*}=\left(\Lambda_{m}+\lambda\right)^{*} \cdots\left(\Lambda_{1}+\lambda\right)^{*}+\sum_{|I| \leq m-1} a_{I}^{0^{*}}(\Lambda+\lambda)_{I}^{*}
$$

For each factor in (3.4) we first shall prove the following inequality: For $s \in \mathbf{N}$ there exists $\lambda_{s} \in \mathbf{N}$ such that for all $\lambda \geq \lambda_{s}$ and $v \in C_{0}^{\infty}((0, T) \times M)$ 
we have

$$
\lambda\|v\|_{L^{2}\left([0, T] ; H_{-s}(M)\right)}^{2} \leq 2\left\|\left(\Lambda_{k}+\lambda\right)^{*} v\right\|_{L^{2}\left([0, T] ; H_{-s}(M)\right)}^{2} .
$$

To prove this, let $\left(\tilde{\Omega}, \theta_{1}, \ldots, \theta_{n-1}\right)$ be a local chart corresponding to one of a finite number of coordinate patches $\left\{U_{j}\right\}$ covering $M$, and let $\left\{\varphi_{j}\right\}$ be a square partition of unity subordinated to the $\left\{U_{j}\right\}$. We use the Sobolev norm

$$
\|v\|_{H_{-s}(M)}^{2}=\sum_{j=1}^{N}\left\|\varphi_{j} v\right\|_{H_{-s}\left(\mathbf{R}_{\theta}^{n-1}\right)}^{2},
$$

and observe that

$$
\begin{aligned}
-t \partial_{t}\left\|\varphi_{j} v\right\|_{-s}^{2}= & 2 \operatorname{Re}\left\langle\left(\Lambda_{k}+\lambda\right) * \varphi_{J} v, \varphi_{j} v\right\rangle_{-s}+2(1-\lambda)\left\|\varphi_{j} v\right\|_{-s}^{2} \\
& +2 \operatorname{Re}\left\langle L_{k}^{*} \varphi_{j} v, \varphi_{J} v\right\rangle_{-s} .
\end{aligned}
$$

The hyperbolicity condition implies that the principal symbol of $L_{k}, i l_{k}$, is purely imaginary, and so we may estimate the last term by $C_{1}\left\|\varphi_{j} v\right\|_{-s}^{2}$. Noting that the commutator $\left[\left(\Lambda_{k}+\lambda\right)^{*}, \varphi_{j}\right]$ is of order 0 , after summing over $j$ and integrating from 0 to $T$, we obtain

$$
(2 \lambda-C) \int_{0}^{T}\|v\|_{H_{-s}(M)}^{2} d t \leq 2 \int_{0}^{T}\left\|\left(\Lambda_{k}+\lambda\right) * v\right\|_{H_{-s}(M)}^{2} d t .
$$

Inequality (3.5) then follows for $\lambda_{s} \geq C$.

Rewriting the factorization (3.4) and using inequality (3.5) allows us to absorb terms of length $m-1$ to yield for $\lambda$ large enough

$$
\begin{aligned}
\sum_{|I|=m-1}\left\|(\Lambda+\lambda)_{I}^{*} v\right\|_{L^{2}\left(H_{-s}\right)}^{2} & \leq\left\|Q_{\lambda}^{*} v\right\|_{L^{2}\left(H_{-s}\right)}^{2}+C \sum_{|I| \leq m-2}\left\|(\Lambda+\lambda)_{I}^{*} v\right\|_{L^{2}\left(H_{-s}\right)}^{2} .
\end{aligned}
$$

Applying (3.5) and this process repeatedly implies there exists $\lambda_{s} \in \mathbf{N}$ such that for all $\lambda \geq \lambda_{s}$ and all $v \in C_{0}^{\infty}((0, T) \times M)$ we have

$$
\|v\|_{L^{2}\left([0, T] ; H_{-s}(M)\right)}^{2} \leq\left\|Q_{\lambda}^{*} v\right\|_{L^{2}\left([0, T] ; H_{-s}(M)\right)}^{2} .
$$

This proves Lemma 1 .

Inequality (3.6) shows that the linear functional $\left\langle t^{-\lambda} f, v\right\rangle_{L^{2}([0, T] \times M)}$ is bounded in the norm $\left[\int_{0}^{T}\left\|Q_{\lambda}^{*} v\right\|_{H_{-s}(M)}^{2} d t\right]^{1 / 2}$, and so, by the Riesz Theorem, admits the representation $\left\langle u_{0}, Q_{\lambda}^{*} v\right\rangle_{L^{2}\left(H_{-s}\right)}$ where $u_{0}$ is in $L^{2}\left([0, T] ; H_{-s}(M)\right)$. Therefore,

$$
\left\langle t^{-\lambda} f, v\right\rangle_{L^{2}([0, T] \times M)}=\left\langle u_{1}, Q_{\lambda}^{*} v\right\rangle_{L^{2}([0, T] \times M)}
$$


for some $u_{1} \in L^{2}\left([0, T] ; H_{s}(M)\right)$, and $Q_{\lambda} u_{1}=t^{-\lambda} f$ in $\mathscr{D}^{\prime}((0, T) \times M)$. With $u_{s, \lambda}=t^{\lambda} u_{1}$, we have $P u_{s, \lambda}=t^{\lambda} Q_{\lambda} u_{1}=f$ in $\mathscr{D}^{\prime}$ and $u_{s, \lambda}$ is in $L_{-\lambda}^{2}\left([0, T] ; H_{s}(M)\right)$. Choosing $\lambda$ sufficiently large then proves Theorem 1 .

\section{Proof of Theorems 2, 3 and Corollary 3.}

Proof of Lemma 2. Theorem 1 tells us there exists a distribution solution $u_{s^{\prime}, r} \in L_{-r}^{2}\left(H_{s^{\prime}}\right)$ to $P u=f$. We will show that this is indeed the $u_{s^{\prime}, l^{\prime} r}$ needed. From now on call it simply $u$. Clearly $u$ belongs to $L_{-(r-m+1)}^{2}\left(H_{s^{\prime}-m+1}\right)$. Manipulating the equation $P u=f$, one can show by induction on $k$ the following sublemma.

Sublemma 1. For each $k=1, \ldots, m-1$ and each $j=0,1, \ldots, m-k$, there exists $Q_{(m-j-k)}^{k}$, a differential operator on $M$, smooth in $t$, of order $m-j-k$, satisfying

$$
Q_{(0)}^{k} \equiv 1, \quad \sum_{j=0}^{m-k}\left(t \partial_{t}\right)^{j} Q_{(m-j-k)}^{k}\left(t^{-(r-k)} u\right) \in L^{2}\left(H_{s^{\prime}-m}\right) .
$$

Using Sublemma 1 with $k=m-1$ implies $\partial_{t} u \in L_{-(r-m)}^{2}\left(H_{s^{\prime}-m}\right)$. Applying Sublemma 1 repeatedly, by induction one can show $\partial_{t}^{l} u$ is in $L_{-(r-m-l+1)}^{2}\left(H_{s^{\prime}-m-l+1}\right), l=0, \ldots, m-1$. For $l=m$ the result follows from the equation

$$
\left(t \partial_{t}\right)^{m} u=f-\sum_{j=0}^{m-1} P_{m-j}\left(t \partial_{t}\right)^{j} u
$$

Finally, for $l>m$, we operate both sides of the equation above by $\left(t \partial_{t}\right)^{l-m}$ and apply similar techniques. This concludes the proof of Lemma 2.

Proof of Theorem 2. Let $k, s \in \mathbf{N}$ be given, and choose $l^{\prime}, s^{\prime}$, and $r$ such that $l^{\prime}>k+1, s^{\prime} \geq s+l^{\prime}+m-1$, and $r \geq m+l^{\prime}$. Denote the solution from Lemma 2 by $u_{s, k}$. In particular, we have $t^{-1} \partial_{t}^{j} u_{s, k} \in L^{2}\left(H_{s}\right)$ for $0 \leq j \leq l^{\prime}$. Since $l^{\prime}$ was chosen appropriately large, Sobolev's Embedding Theorem implies that our solution is in $C^{k}\left((0, T) ; H_{s}(M)\right)$, and it is easy to see that $\lim _{t \rightarrow 0+} \partial_{t}^{J} u_{s, k}=0,0 \leq j \leq k$. Thus, for any $T_{0} \in(0, T)$, $u_{s, k} \in C^{k}\left(\left[0, T_{0}\right] ; H_{s}(M)\right)$ and $P u_{s, k}=f$ pointwise in $\left[0, T_{0}\right] \times M$. This proves the theorem.

Proof of Theorem 3. Theorem 2 provides a solution in $C^{k}\left(\left[0, T_{0}\right] ; H_{s}(M)\right)$ flat to order $k$. Choosing $k$ large enough and applying 
the uniqueness result of Alinhac and Baouendi (Theorem 2 of [3]), gives

$$
u_{s, k}=u_{s, k+1}=\cdots=u_{s, \infty} \in C^{\infty}\left(\left[0, T_{1}\right] ; H_{s}(M)\right)
$$

with $T_{1} \in\left(0, T_{0}\right]$ and $u_{s, \infty}$ flat at $t=0$. Since we are free to pick $s$ larger and larger, we have by Sobolev's Embedding Theorem that $u=u_{s, \infty}$ is actually in $C^{\infty}\left(\left[0, T_{1}\right] \times M\right)$. Now let $0<T \leq \infty$ and $0<\varepsilon<T_{1}$. Since $P$ is strictly hyperbolic with respect to $\{t=\varepsilon\}$, there exists a unique $u_{\varepsilon}$ in $C^{\infty}([\varepsilon, T] \times M)$ satisfying $P u_{\varepsilon}=f$ in $[\varepsilon, T] \times M$ and $\left.\partial_{t}^{J} u_{\varepsilon}\right|_{t=\varepsilon}=\left.\partial_{t}^{J} u\right|_{t=\varepsilon}$, $0 \leq j \leq m-1$. The result of Theorem 3 then follows by the uniqueness of the solution in $\left[\varepsilon, T_{1}\right] \times M$.

Proof of Corollary 3. By techniques similar to those of K. Yamamoto [10], we can use the Levi-type condition (1.5) to show the following factorization for $P$ :

$$
P=\sum_{|I| \leq m} \gamma_{I}^{0} A_{I}, \quad \text { with } \gamma_{I}^{0} \in C^{\infty}\left([0, T] ; \mathscr{L}^{0}(M)\right)
$$

and $A_{j}$ defined to be $\Lambda_{l}$ for every $j$ satisfying $m_{1}+\cdots+m_{l-1}+1 \leq j \leq$ $m_{1}+\cdots+m_{l}$. Combining this factorization with the assumption on the principal symbol, (1.4), implies actually

$$
P=\Lambda_{1}^{m_{1}} \Lambda_{2}^{m_{2}} \cdots \Lambda_{r}^{m_{r}}+\sum_{|I| \leq m-1} \gamma_{I}^{0}\left(t, \theta, D_{\theta}\right) A_{I},
$$

or, after manipulating commutators,

$$
P=\Lambda_{1}^{m_{1}} \cdots \Lambda_{r}^{m_{r}}+\sum_{|I| \leq m-1} A_{I} \tilde{\gamma}_{I}^{0} .
$$

This factorization is all that is needed now to get the conclusions of Theorems 1 and 2. For uniqueness, we see that each factor has the form $\Lambda_{j}=t \partial_{t}-L_{j}$, and by virtue of the fact that the principal symbol of $L_{J}$ has no real part, one has the estimate

$$
\operatorname{Re}\left\langle L_{J} v, v\right\rangle_{L^{2}(M)} \geq-C\|v\|_{L^{2}(M)}^{2} \text { for all } v \in C^{1}\left([0, T] ; H_{2}(M)\right) .
$$

Let $T_{1} \in(0, T)$. For functions $u \in C^{k}\left([0, T] ; H_{2}(M)\right)$, flat to order $k$ (with $k$ large enough), by setting $v=t^{-\gamma} u$ and using (4.1), one can show for $\gamma$ large enough that

$$
\gamma\left\|t^{-\gamma} u\right\|_{L^{2}\left(\left[0, T_{1}\right] ; L^{2}(M)\right)} \leq 2\left\|t^{-\gamma} \Lambda_{j} u\right\|_{L^{2}\left(\left[0, T_{1}\right] ; L^{2}(M)\right)}
$$

for each $j=1, \ldots, r$. This, the factorization, and the same method used in proving Lemma 1 yields for $k$ and $\gamma$ sufficiently large

$$
\left\|t^{-\gamma} u\right\|_{L^{2}\left(L^{2}\right)} \leq\left\|t^{-\gamma} P u\right\|_{L^{2}\left(L^{2}\right)} .
$$


With the desired uniqueness for functions sufficiently flat, we may now conclude as in the proof of Theorem 3.

\section{Proof of Theorems 4 and 5.}

Proof of Theorem 4. To prove this theorem we shall first need an energy estimate similar to that of Lemma 1.

LEMMA 3. With the same assumptions as in Theorem 4 (except here we assume $0<T<\infty)$, given any $s \in \mathbf{N}$, there exists $\lambda_{s} \in \mathbf{N}$ such that for all $\lambda \geq \lambda_{s}$ and all $v \in C_{0}^{\infty}((0, T) \times \Omega \times M)$ we have

$$
\|v\|_{L^{2}\left([0, T] ; H_{-s}(\Omega \times M)\right)} \leq\left\|t^{-1 / 2} Q_{\lambda}^{*} v\right\|_{L^{2}\left([0, T] ; H_{-s}(\Omega \times M)\right)} .
$$

Proof of Lemma 3. Since $P$ satisfies (2.1) with $m=2$, we may write

$$
P=\left(t \partial_{t}\right)^{2}+2 Q_{1}\left(t, x, \theta, t \partial_{x}, \partial_{\theta}\right) t \partial_{t}+Q_{2}\left(t, x, \theta, t \partial_{x}, \partial_{\theta}\right),
$$

with $Q_{1}$ and $Q_{2}$ first and second order differential operators respectively. The condition (2.2) says we may rewrite the operator as

$$
P=\left(t \partial_{t}+Q_{1}\right)^{2}-\tilde{Q},
$$

where $\tilde{Q}$ is a second order differential operator in $t \partial_{x}$ and $\partial_{\theta}$, whose principal symbol $\tilde{q}$, considered as a function of $t \xi$ and $\eta$, is elliptic and real. Moreover, the principal symbol of $\tilde{Q}^{*}$ satisfies $\tilde{q}^{*}=\tilde{q}<0$.

We shall first prove local estimates and then derive the global estimate (5.1). For a local chart $\left(\tilde{\Omega}, \theta_{1}, \ldots, \theta_{n-1}\right)$, then, let $w$ be in $C_{0}^{\infty}((0, T) \times \Omega \times \tilde{\Omega})$. Since $-\tilde{q}^{*}$ is positive and elliptic, we have by homogeneity that

$$
-\tilde{q}^{*}(t, x, \theta, t \xi, \eta) \geq C\left(|t \xi|^{2}+|\eta|^{2}\right)
$$

for all $(t, x, \theta, t \xi, \eta) \in[0, T] \times\left(T^{*}(\Omega \times \tilde{\Omega}) \backslash 0\right)$. Hence, one may associate with this a definite positive hermitian quadratic form $-\tilde{q}^{*}$, whose value on $\left(t \nabla_{x} w, \nabla_{\theta} w\right)$ has the coercivity

$$
-\tilde{q}^{*}(\nabla w) \geq C\left(\left|t \nabla_{x} w\right|^{2}+\left|\nabla_{\theta} w\right|^{2}\right) .
$$

Recall that for $\gamma \in \mathbf{N}$, the operator $Q_{\gamma}$ is just $P\left(t, x, \theta, t \partial_{t}+\gamma, t \partial_{x}, \partial_{\theta}\right)$. We may therefore write

$$
Q_{\gamma}^{*}=\left(t \partial_{t}+1-\gamma-Q_{1}^{*}\right)^{2}-\tilde{Q}^{*}
$$


Let us now define the function

$$
\begin{aligned}
z(t, w)= & \|w\|_{H_{-s}\left(\mathbf{R}_{x, \theta}^{n+\nu-1}\right)}^{2+1}+\left\|\left(t \partial_{t}+1-\gamma-Q_{1}^{*}\right) w\right\|_{H_{-s}\left(\mathbf{R}_{x, \theta}^{n+\nu-1}\right)}^{2} \\
& +\int_{\Omega \times \tilde{\Omega}}-\tilde{q}^{*}\left(\nabla \Lambda_{-s}^{\prime} w\right) d x d \theta
\end{aligned}
$$

with $\Lambda_{-s}^{\prime}$ some proper operator with symbol $\left(1+|\xi|^{2}+|\eta|^{2}\right)^{-s / 2}$. We claim there is a constant $C>0$ such that if $\gamma \geq 2$ we have the following estimate:

$$
-t \partial_{t} z(t, w) \leq C(\gamma-1)^{2} z(t, w)+\left\|Q_{\gamma}^{*} w\right\|_{H_{-s}\left(\mathbf{R}_{x, \theta}^{n+\nu-1}\right)}^{2} .
$$

Using the coercivity (5.2), we obtain after lengthy computations

$$
\begin{aligned}
& -t \partial_{t} \int_{\Omega \times \tilde{\Omega}}-\tilde{q}^{*}\left(\nabla \Lambda_{-s}^{\prime} w\right) d x d \theta \\
& \quad \leq 2 \operatorname{Re}\left\langle t \partial_{t} w, \tilde{Q}^{*} w\right\rangle_{-s}+c \int_{\Omega \times \tilde{\Omega}}-\tilde{q}^{*}\left(\nabla \Lambda_{-s}^{\prime} w\right) d x d \theta \\
& \quad+C\left\|\left(t \partial_{t}+1-\gamma-Q_{1}^{*}\right) w\right\|_{-s}^{2}+C(\gamma-1)^{2}\|w\|_{-s}^{2} .
\end{aligned}
$$

For the other two pieces in (5.4), we observe that since the principal symbol of $Q_{1}^{*}$ is purely imaginary we have

$$
-t \partial_{t}\|v\|_{-s}^{2} \leq-2 \operatorname{Re}\left\langle\left(t \partial_{t}+1-\gamma-Q_{1}^{*}\right) v, v\right\rangle_{-s}+(C-2 \gamma)\|v\|_{-s}^{2}
$$

for all $v \in C_{0}^{\infty}((0, T) \times \Omega \times \tilde{\Omega})$. Applying this estimate for $v=w, v=$ $\left(t \partial_{t}+1-\gamma-Q_{1}^{*}\right) w$, and using (5.4) and (5.6) yields

$$
\begin{aligned}
-t \partial_{t} z \leq & -2 \operatorname{Re}\left\langle\left(t \partial_{t}+1-\gamma-Q_{1}^{*}\right)^{2} w,\left(t \partial_{t}+1-\gamma-Q_{1}^{*}\right) w\right\rangle_{-s} \\
& +C(\gamma-1)^{2} z+2 \operatorname{Re}\left\langle\tilde{Q}^{*} w, t \partial_{t} w\right\rangle_{-s} .
\end{aligned}
$$

Since $Q_{1} \tilde{Q}^{*}+\tilde{Q} Q_{1}^{*}$ is a second order differential operator in $t \partial_{x}$ and $\partial_{\theta}$, from the coercivity (5.2), we can estimate the last term by

$$
\begin{aligned}
& 2 \operatorname{Re}\left\langle\tilde{Q}_{1}^{*} w,\left(t \partial_{t}+1-\gamma-Q_{1}^{*}\right) w\right\rangle_{-s} \\
& \quad+C(\gamma-1)\left[\|w\|_{-s}^{2}+\int_{\Omega \times \tilde{\Omega}}-\tilde{q}^{*}\left(\nabla \Lambda_{-s}^{\prime} w\right) d x d \theta\right] .
\end{aligned}
$$

Hence, by (5.3) and (5.4), the inequality (5.5) follows immediately.

The local estimate (5.5) can now be used to derive (5.1) in the following way. For $w \in C_{0}^{\infty}((0, T) \times \Omega \times M)$, we use the Sobolev norm

$$
\|w\|_{H_{-s}(\Omega \times M)}^{2}=\sum_{k=1}^{N}\left\|\varphi_{k} w\right\|_{-s}^{2}, \quad \sum_{k=1}^{N} \varphi_{k}^{2}=1 \quad \text { on } M
$$


and use (5.5) for each $z_{k}\left(t, \varphi_{k} w\right)$. Setting $\Phi=\sum_{k=1}^{N} z_{k}\left(t, \varphi_{k} w\right)$, observing that

$$
\left\|Q_{\gamma}^{*} \varphi_{k} w\right\|_{-s}^{2} \leq \tilde{C}(\gamma-1)^{2} \Phi+2\left\|\varphi_{k} Q_{\gamma}^{*} w\right\|_{-s}^{2},
$$

and summing over $k$, we have

$$
-t \partial_{t} \Phi \leq C(\gamma-1)^{2} \Phi+2\left\|Q_{\gamma}^{*} w\right\|_{H_{-s}(\Omega \times M)}^{2} .
$$

If we let $C_{\gamma}=(C / 2)(\gamma-1)^{2}$, this Gronwall-type inequality implies

$$
t^{2 C_{\gamma}} \Phi \leq 2 \int_{0}^{T} \tau^{2 C_{\gamma}-1}\left\|Q_{\gamma}^{*} w\right\|_{H_{-s}(\Omega \times M)}^{2} d \tau,
$$

and from (5.4) it follows that

$$
\left\|t^{C_{\gamma}} w\right\|_{L^{2}\left([0, T] ; H_{-,}(\Omega \times M)\right)}^{2} \leq 2 T\left\|t^{-1 / 2} t^{C_{\gamma}} Q_{\gamma}^{*} w\right\|_{L^{2}\left([0, T] ; H_{-,}(\Omega \times M)\right)}^{2} .
$$

By (5.3), one can easily check that $t^{C_{\gamma}} Q_{\gamma}^{*} w=Q_{\left(\gamma+C_{\gamma}\right)}^{*}\left(t^{\left.C_{\gamma} w\right)}\right.$. Thus if $v \in C_{0}^{\infty}((0, T) \times \Omega \times M)$, we set $w=t^{-C_{\gamma} v}$ to obtain for all $\gamma \geq 2$

$$
\|v\|_{L^{2}\left(H_{-s}\right)}^{2} \leq 2 T\left\|t^{-1 / 2} Q_{\left(\gamma+C_{\gamma}\right)}^{*}(v)\right\|_{L^{2}\left(H_{-s}\right)}^{2} .
$$

Since $C_{\gamma}=(C / 2)(\gamma-1)^{2}$, this inequality with $T<1 / 2$ and $\lambda_{s}=2$ $+(C / 2)$ implies (5.1). Hence Lemma 3 has been proved.

If $f$ has compact support in the $x$-directions, then the estimate (5.1) shows that there exists $u_{0} \in L^{2}\left([0, T] ; H_{s}\left(\Omega^{\prime} \times M\right)\right), \Omega^{\prime} \Subset \Omega$, such that $Q_{\lambda}\left(t^{-1 / 2} u_{0}\right)=t^{-\lambda} f$ in $\mathscr{D}^{\prime}\left((0, T) \times \Omega^{\prime} \times M\right), \lambda$ large. But this gives a distribution solution $u=t^{[\lambda-(1 / 2)]} u_{0}$ to $P u=f$. With $T_{0} \in(0, T)$ and $k \in \mathbf{N}$, using the same methods as in the proof of Theorem 2 we get a $C^{k}$ solution to $P u=f$, flat to the order $k$ at $t=0$. We now apply another uniqueness result of Alinhac and Baouendi (Theorem 4' of [3]). As in the proof of Theorem 3, then, with $0<T \leq \infty, \Omega$ replacing $\Omega^{\prime}$, and dropping the support restriction on $f$, we see that the conclusion of Theorem 4 follows.

Proof of Theorem 5. With $Q_{\lambda}^{*}=-t \partial_{t}-1+\lambda-\sum_{l} t \partial_{x_{l}} A_{l}+\Lambda^{*}$, since $\Lambda+\Lambda^{*}$ is of order 0 (hyperbolicity condition), we obtain a similar estimate to (5.1). We simply conclude now as in the proof of Theorem 4, except here we use still another uniqueness result of Alinhac and Baouendi-namely Theorem 4 of [3]. This completes the proof.

Acknowledgment. The author is grateful to M. S. Baouendi for his support and encouragement. 


\section{REFERENCES}

[1] S. Alinhac, Problemes de Cauchy pour des operateurs singuliers, Bull. Soc. Math. France, 102 (1974), 289-315.

[2] __ Systemes hyperbolique singuliers, Asterique, 19 (1974), 3-25.

[3] S. Alinhac and M. S. Baouendi, Uniqueness for the characteristic Cauchy problem and strong unique continuation for higher order partial differential inequalities, Amer. J. Math., 102 (1980), 179-217.

[4] M. S. Baouendi and C. Goulaouic, Cauchy problems with characteristic initial hypersurface, Comm. Pure Appl. Math., 26 (1973), 455-475.

[5] L. Hörmander, Pseudo-differential operators and hypoelliptic equations, Proc. Sympos. Pure Math., Vol. 10, Amer. Math. Soc., Providence, R. I., 1968, 138-183.

[6] L. Nirenberg, Pseudo-differential operators, Proc. Sympos. Pure Math., Vol. 16, Amer. Math. Soc., Providence, R. I., 1970, 149-167.

[7] _ Lectures on Linear Partial Differential Equations, C.B.M.S., Amer. Math. Soc., 17 (1972).

[8] G. Roberts, Uniqueness in the Cauchy problem for characteristic operators of Fuchsian type, J. Differential Equations, 38 no. 3 (1980), 374-392.

[9] F. Treves, Introduction to Pseudodifferential and Fourier Integral Operators, Vol. 1, Plenum Press, New York, 1980.

[10] K. Yamamoto, The Cauchy problem for some class of hyperbolic differential operators with variable multiple characteristics, J. Math. Soc. Japan, 31 (1979), 481-502.

Received April 12, 1983

OKLahoma State University

STILLWATER, OK 74078 


\title{
PACIFIC JOURNAL OF MATHEMATICS EDITORS
}

\author{
Donald BABBITt (Managing Editor) \\ University of California \\ Los Angeles, CA 90024 \\ J. DugunduI \\ University of Southern California \\ Los Angeles, CA 90089-1113 \\ R. FINN \\ Stanford University \\ Stanford, CA 94305 \\ HermanN FlaschKa \\ University of Arizona \\ Tucson, AŻ 85721
}

C. C. Moore

University of California

Berkeley, CA 94720

ARTHUR Ogus

University of California

Berkeley, CA 94720

Hugo Rossi

University of Utah

Salt Lake City, UT 84112

H. SAMELSON

Stanford University

Stanford, CA 94305

ASSOCIATE EDITORS
R. ARENS
E. F. BECKENBACH
B. H. NEUMANN
F. WOLF
K. YoSHIDA (1906-1982)

\section{SUPPORTING INSTITUTIONS}

$\begin{array}{ll}\text { UNIVERSITY OF ARIZONA } & \text { UNIVERSITY OF OREGON } \\ \text { UNIVERSITY OF BRITISH COLUMBIA } & \text { UNIVERSITY OF SOUTHERN CALIFORNIA } \\ \text { CALIFORNIA INSTITUTE OF TECHNOLOGY } & \text { STANFORD UNIVERSITY } \\ \text { UNIVERSITY OF CALIFORNIA } & \text { UNIVERSITY OF HAWAII } \\ \text { MONTANA STATE UNIVERSITY } & \text { UNIVERSITY OF TOKYO } \\ \text { UNIVERSITY OF NEVADA, RENO } & \text { UNIVERSITY OF UTAH } \\ \text { NEW MEXICO STATE UNIVERSITY } & \text { WASHINGTON STATE UNIVERSITY } \\ \text { OREGON STATE UNIVERSITY } & \text { UNIVERSITY OF WASHINGTON }\end{array}$

The Supporting Institutions listed above contribute to the cost of publication of this Journal, but they are not owners or publishers and have no responsibility for its content or policies.

Mathematical papers intended for publication in the Pacific Journal of Mathematics should be in typed form or offset-reproduced (not dittoed), double spaced with large margins. Please do not use built up fractions in the text of the manuscript. However, you may use them in the displayed equations. Underline Greek letters in red, German in green, and script in blue. The first paragraph must be capable of being used separately as a synopsis of the entire paper. In particular it should contain no bibliographic references. Please propose a heading for the odd numbered pages of less than 35 characters. Manuscripts, in triplicate, may be sent to any one of the editors. Please classify according to the scheme of Math. Reviews, Index to Vol. 39. Supply name and address of author to whom proofs should be sent. All other communications should be addressed to the managing editor, or Elaine Barth, University of California, Los Angeles, California 90024.

There are page-charges associated with articles appearing in the Pacific Journal of Mathematics. These charges are expected to be paid by the author's University, Government Agency or Company. If the author or authors do not have access to such Institutional support these charges are waived. Single authors will receive 50 free reprints; joint authors will receive a total of 100 free reprints. Additional copies may be obtained at cost in multiples of 50 .

The Pacific Journal of Mathematics is issued monthly as of January 1966. Regular subscription rate: $\$ 190.00$ a year (5 Vols., 10 issues). Special rate: $\$ 66.00$ a year to individual members of supporting institutions.

Subscriptions, orders for numbers issued in the last three calendar years, and changes of address should be sent to Pacific Journal of Mathematics, P.O. Box 969, Carmel Valley, CA 93924, U.S.A. Old back numbers obtainable from Kraus Periodicals Co., Route 100, Millwood, NY 10546.

The Pacific Journal of Mathematics at P.O. Box 969, Carmel Valley, CA 93924 (ISSN 0030-8730) publishes 5 volumes per year. Application to mail at Second-class postage rates is pending at Carmel Valley, California, and additional mailing offices. Postmaster: Send address changes to Pacific Journal of Mathematics, P.O. Box 969, Carmel Valley, CA 93924.

PUBLISHED BY PACIFIC JOURNAL OF MATHEMATICS, A NON-PROFIT CORPORATION

Copyright $@ 1984$ by Pacific Journal of Mathematics 


\section{Pacific Journal of Mathematics}

\section{Vol. 115, No. $2 \quad$ October, 1984}

Ersan Akyildiz, Gysin homomorphism and Schubert calculus ...........257

Marilyn Breen, Clear visibility and unions of two starshaped sets in the

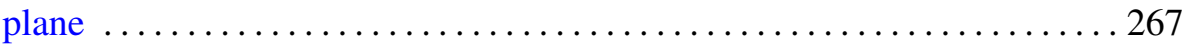

Robert F. Brown, Retraction methods in Nielsen fixed point theory . ......277

Herbert Busemann and Bhalchandra B. Phadke, A general version of Beltrami's theorem in the large ............................... 299

Gerald Arthur Edgar and Robert Francis Wheeler, Topological properties of Banach spaces ............................... 317

Yaakov Friedman and Bernard Russo, Conditional expectation without

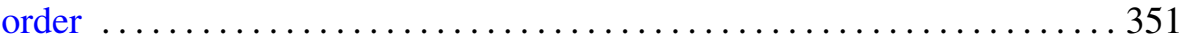

Robert Allen Goggins, Cobordism of manifolds with strong almost tangent

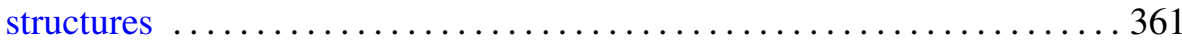

Mike Hoffman, Noncoincidence index of manifolds . . . . . . . . . . . . . 373

William H. Julian, $\varepsilon$-continuity and monotone operations $\ldots \ldots \ldots \ldots 385$

Gerasimos E. Ladas, Y. G. Sficas and I. P. Stavroulakis, Nonoscillatory

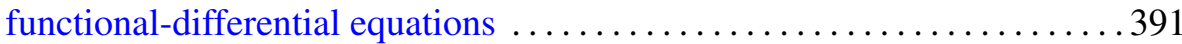

Arnold William Miller and Karel Libor Prikry, When the continuum has

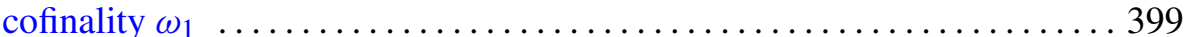

Jean-Leah Mohrherr, Density of a final segment of the truth-table

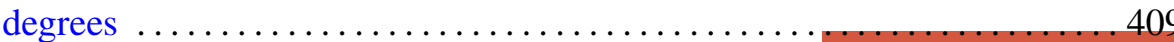

Carl Norman Mutchler, The flat Cauchy problem for radially hyperbolic operators from a characteristic manifold of high codimension ...

Kenji Nakagawa, On the orders of automorphisms of a closed Riemann surface

W. Ricker, Representation of vector-valued functions by Laplace transforms

Jorge Donato Samur, On semigroups of convolution operators in Hilbert

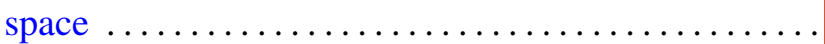

Joseph Gail Stampfli, One-dimensional perturbations of operators 481 Andrew George Earnest and John Sollion Hsia, Correction to: "Spinor norms of local integral rotations. II" 